\title{
Cervix and breast cancers in Oujda city in Eastern Morocco: Determinants and risk factors
}

\author{
Abdellatif Maamri ${ }^{1}$, Mohamed El Hfid ${ }^{2}$, Abdelhafid Chafi $^{3}$, Abdesslam Boutayeb $^{4 *}$ \\ ${ }^{1}$ Health Career Training Institute \& Faculty of Sciences, Oujda, Morocco; abdellatifm@hotmail.com \\ ${ }^{2}$ Faculty of Medicine and Pharmacy, University Mohamed I, Oujda, Morocco; melhfid@yahoo.fr \\ ${ }^{3}$ Department of Biology, Faculty of Science, Oujda, Morocco; chafihafid@yahoo.fr \\ ${ }^{4}$ Department of Mathematics, Faculty of Science, URAC04, Oujda, Morocco; ${ }^{*}$ Corresponding Author: x.boutayeb@menara.ma
}

Received 11 October 2011; revised 27 November 2011; accepted 31 December 2011

\section{ABSTRACT}

A retrospective study was conducted in 2008 on 681 in Eastern Morocco in order to estimate the incidence of cervical and breast cancer, and to determine the social determinants and risk factors associated with these two types of cancer. Another goal of the study was to give health decision makers evidence of the necessity for early diagnosis and treatment. A questionnaire was used before the clinical gynecological examination and sampling cell for the Pap smear. Any woman with a Pap smear suspect had to do a biopsy at the reference centre. Physicians, obstetricians and midwives in the consultation process did gynecological examination and breast palpation. Patients with a suspicious nodule underwent ultrasound and mammography by radiologists on site. Gynecological samples were carried out systematically to all ever-married women to detect cancer of the cervix. Software Excel and SPSS 17 were used for data processing. Patients with high social status were more susceptible to develop breast cancer whereas women with cervical cancer were poor, belonging to a class with a low marital status of widowed or divorced having been married at an early age. The majority of women were married (81\%), poor $(80 \%)$ and illiterate (66\%). Breastfeeding women were less affected by breast cancer than women not breastfeeding. For cervical cancer, the difference was not statistically significant. Women using contraceptives were vulnerable to both breast and cervical cancer. For cervical cancer, over $15 \%$ of women with a family history were screened positive. No relationship was found between breast cancer and family history. Taking into account regional particularities, we stress the importance of social determinants and risk factors and show that our results are consistent with those published by other researchers. Breast and cervical cancers constitute a real challenge in Morocco. Their socioeconomic burden can be reduced by early detection and treatment. The delayed diagnosis complicates the task both in terms of survival and cost of treatment. Consequently, Moroccan health authorities are urged to adopt preventive and cost effective strategies.

Keywords: Cervical; Breast; Cancer; Determinants; Risk; Factor; Strategy; Morocco

\section{INTRODUCTION}

The Cancer is one of the leading causes of death worldwide $[1,2]$. The global mortality from cancer exceeds that of AIDS, tuberculosis and malaria combined. According to the American Cancer Society, nearly eight million people die each year from cancer in the world.

The incidence of cancer was multiplied by four in 10 years among women 35 - 45 years old. Breast and cervical cancer represent successively the first and second cancer killer of women. Worldwide, more than 1.05 million new cases of breast cancer occur each year, including over 580,000 in developed countries (Western Europe and North America) where it is more common than in Africa or Asia. This type of cancer is responsible for more than 400,000 deaths of women each year.

Similarly, the epidemiological data on cervix cancer released by the National Institute of Cancer in France (INCa) [3], showed that in 2005, cervix cancer was the second female cancer worldwide in terms of incidence $(500,000$ new cases) and mortality (260,000 deaths), with $95 \%$ of deaths occurring in developing countries. Cervical cancer represents $10 \%$ of female deaths from cancer and affects mainly pre-menopausal women (40 - 50) [4].

In Morocco, cancer is a major public health problem [5]. According to data from the Cancer Registry of the 
Greater Casablanca, the national annual incidence of cancer is estimated at 101.7 new cases per 100,000 inhabitants. This would correspond to 30,500 new cases of cancer each year. The Registry also states that, among all types of cancer, breast cancer and cervical cancer represent respectively $36.12 \%$ and $12.82 \%$ [6]. Similarly, in the East Region, the Regional Centre of Oncology Hassan II, indicated a rate of $31.2 \%$ for breast cancer and $8.71 \%$ for cancer of the cervix during the year 2007 [7].

Although breast and cervical cancers are the most deadly for women, they are more easily cured when early detected and treated. It is estimated that their burden can be reduced by one third by early diagnosis and treatment. For this reason and, as part of the national effort to raise awareness, campaigns are organized in some regions of Morocco, hoping to create a climate of sensitization about the disease and hence to promote early detection.

\section{METHOD}

\subsection{Study Population}

This is an epidemiological study conducted in 2008 among women in the prefecture of Oujda. The choice of health centres where women were recruited was planned in such a way as to cover the whole territory of the prefecture. Then, in order to get the best estimate of breast and cervical cancers in the Eastern region, a sample was formed with 681 women recruited randomly at the selected health centres. A thorough examination was carried out using a questionnaire before the clinical gynaecological examination and sampling cell for Pap smear. Any woman with a suspicious Pap smear had to be biopsied at the centre of reference. If histology confirmed the cytology, the patient was addressed to the cancer centre of Oujda.

\subsection{Data Collection and Statistical Analysis}

At the centre of Reference, physicians, obstetricians and midwives had to carry out gynaecological examination for women and palpation of breasts. Gynaecological samples were taken in a systematic way from any unmarried woman in order to detect cancer of the cervix. The blades were mounted, stained and examined on site by a pathologist. The Pap smear (FCV) was achieved using a spatula of Ayre by collecting cells at the exo-endocervical junction. The cells were spread on slides, dried, fixed and stained with the method of Shorr and Harris. The reading was done according to the nomenclature of Bethesda after mounting on a slide. Three to four biopsies generally small, preserved in Bouin's fluid, arrived at the laboratory. After fixation, dehydration and waxing fragments; sections were spread on slides and stained with eosin hyalin. Mammograms and scans were also performed for women with suspected breast nodules.
A computer program was used to deal with all the information provided by the questionnaire completed by doctors for every woman. The software Excel and SPSS 17 were used for data processing.

\subsection{Ethical Approval and Patients Consent}

The study respected the ethical rules for medical research involving human subjects as stipulated by the WMA Declaration of Helsinki. According to national and local legislation concerning health research, we needed to obtain approval from the highest regional health authority. We effectively received ethics approval from the scientific board which acts as the ethic committee on behalf of the Regional Health Direction (RHD) and its institutional board. The research team explained the details of the study including: the reason why the study was needed, what the researchers hoped to show and all risks and expected benefits for the patient. Data was treated anonymously with agreement of all patients who were informed on the study protocol, gave their consent and decided to participate voluntary.

\section{RESULTS}

The most dominant age group in our sample was formed by women aged between 41 and 50 years (40\%) followed by the age class from 31 to 40 years (31\%). The age categories [51,60], less than 30 and more than 60 represented respectively 16\%, 9\% and 4\% (Figure 1(a)).

Our sample was characterized by a significant proportion of married women (81\%). Widows, divorced and singles sharing the remainder proportion almost equally (6\% 7\%) (Figure1(b)). Most women in the sample were poor with a percentage of about $80 \%$. Craft functions represented $10 \%$ and civil servants $9 \%$ (Figure 1(c)). The patients were mostly illiterate (66\%), followed by those with a preschool level (15\%), secondary level (9\%), primary level (7\%) and university (3\%) (Figure 1(d)).

As shown in Figure 2(a) below, breastfeeding women were less affected by breast cancer than women not breastfeeding ( $p<0.0001)$. Over $10 \%$ of non-breastfeeding women suffer from breast cancer. For cervical cancer, no statistically significant difference was reported ( $p>0.05$ ).

Similarly, women using contraceptive methods as pill or injectable were more vulnerable to breast and/or cervix cancer (Figure 2(b)). Indeed, 8\% of women who developed cervical cancer used injectables and almost 3\% of women with breast cancer used pills.

Nulliparous women had 3\% and 3.30\% of breast and uterine cancer respectively (Figure 3(a)).

Breast cancer occurs particularly beyond 18 years. In opposition, the cervix cancer seems to occur at an early age of marriage (Figure 3(b)). Cancer of the cervix seems to be related to genetics, since over $15 \%$ of women with a family history were screened positive (Figure 3(c)). 
(a)

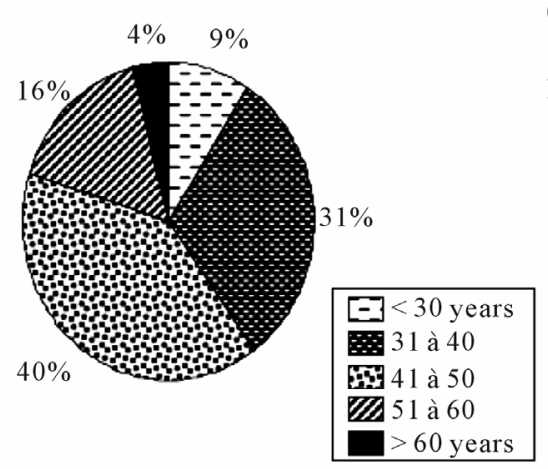

(c)

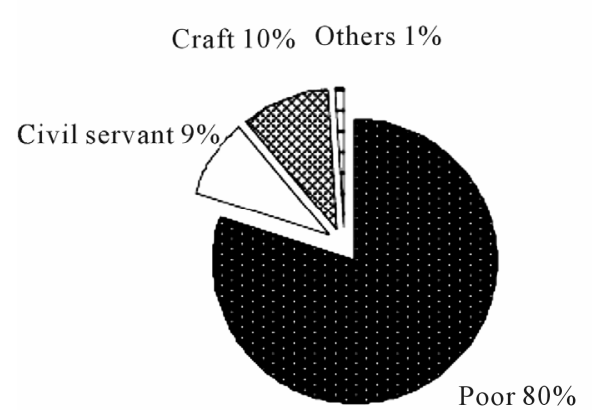

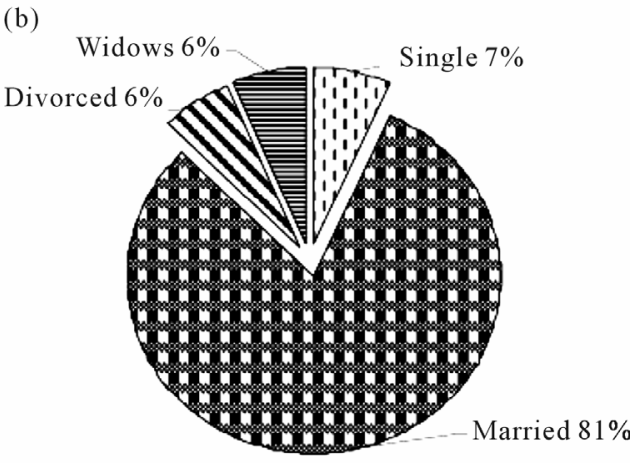

(d)

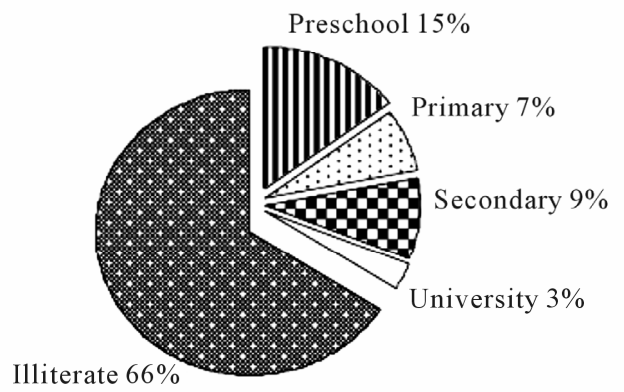

Figure 1. Distribution of women screened by age (a), marital status (b), occupation (c) and level of education (d).

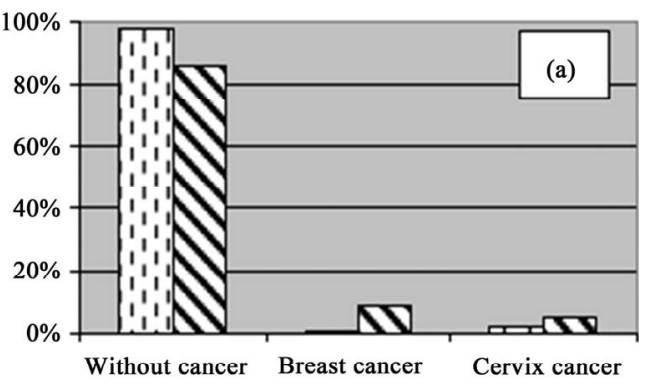

1 Lactating women $\quad \mathbf{W}$ Non lactating women

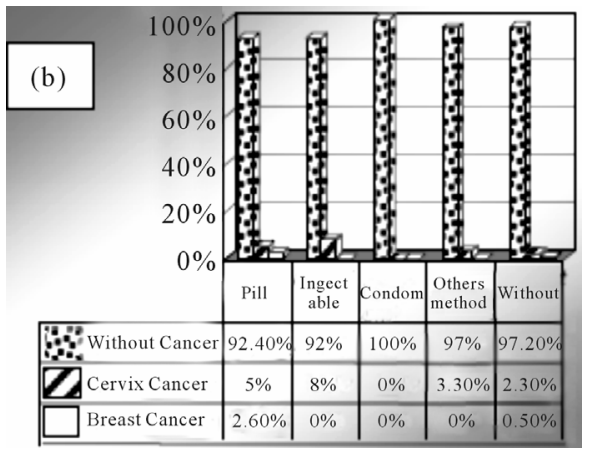

Figure 2. Distribution of of women screened according to breastfeeding (a) and the contraceptive method used (b).

According to our data, breast and cervical cancer affect both poor and civil servant women. However, civil servants are particularly affected by breast cancer (4\%) whereas poor women are more affected by cervical cancer (3.40\%) (Figure 3(d)).

\section{DISCUSSION}

According to the World Health Organisation (WHO), breast and cervical cancer are among the most deadly cancers in the world. However, they are more easily curable when detected early and treated in time, which explains the need for regular campaigns for cancer screenings held in different countries [8].
As part of a policy aiming to reduce the burden of cancer, the Moroccan Health Ministry and the Association Lalla Salma encourage early detection through the organization of campaigns in different regions of Morocco. Indeed, the multiple risk factors, the frequency and severity of these types of cancer in our country suggest the great value of screening.

Reduced risk of death has been demonstrated especially after age 50 years in several countries including France, which has systematized screening [3].

The important Risk factors for these cancers are related to socio-economic factors, reproductive and hormonal factors, but also to environmental factors $[9,10]$. 

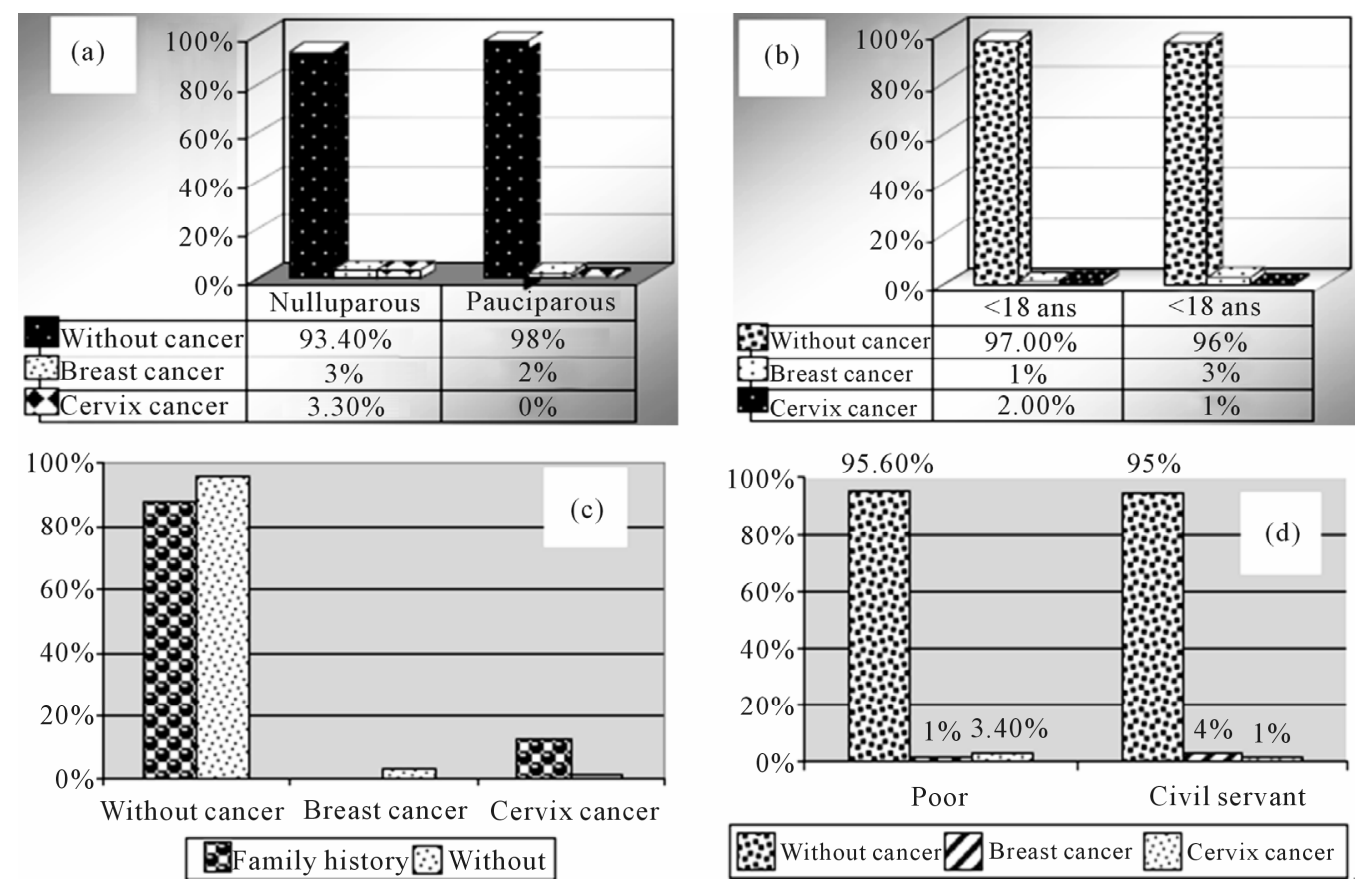

Figure 3. Distribution of women screened according to gesture (a), age of first marriage (b), family history (c) and socioeconomic level (d).

\subsection{Breast Cancer: Social Determinants and Risk Factors}

In our study, we found that the risk of breast cancer was clearly associated with a high socioeconomic level. This is consistent with published literature [8,11]. The difference in incidence may be seen as a direct consequence of the gradient in risk factors between social classes (such as parity, age at first menstruation and menopause $\cdots$ ). Indeed, women with high socioeconomic status have generally low parity $(1-2)$, breastfeed only rarely, little or not at all [12].

\subsubsection{Breastfeeding and Lifestyle}

According to the INCa [3], breastfeeding exclusively and ideally until the age of six months remains one of the absolute recommendations for primary prevention of cancer. This recommendation is consistent with our data since we found a statistically significant difference between nonbreastfeeding and breastfeeding women regarding breast cancer $(\mathrm{p}<0.0001)$.

For Kato et al. [13], in countries with low incidence, increased incidence of breast cancer is the result of westernization of lifestyle on women's reproductive life, diet, physical activity and other factors. It should be recalled that, researchers recognized long ago that early puberty and/or a first pregnancy at a late age and/or absence of breastfeeding are the main risk factors associated with the reproductive life of woman. Usually in developing countries, women are protected by a delayed puberty, an early first birth and high parity which causes often a long pe- riod of lactation. But with the new generations, the behaviours of the reproductive life have evolved in a rapid and significant way: first birth later and later, often not breastfeeding [8].

Taking into account the socio-cultural context of our country, it appears that the age of marriage is becoming increasingly late and the first pregnancy increasingly posponed. Consequently births are delayed and periods of breast-feeding shortened, putting women at a higher risk of cancer [14]. Moreover, the age of puberty is being reached earlier and earlier over the years [15].

\subsubsection{Genetic Effect}

The genetic component may be responsible for breast cancer in one part of the population. For instance, in European populations, approximately $2 \%$ of breast cancer may be due to gene mutations, but the proportion of breast cancer is higher among young people around $10 \%$ below the age of 40 years [16,17].

According to Sasco [18], the most remarkable discoveries of the past 20 years, in terms of etiology of breast cancer, were made in the field of genetics with the roles of two mutations of BRCA1 and BRCA2. Indeed, several studies concur that women with one of these two mutations are $45 \%$ to $80 \%$ at risk of developing breast cancer while in the general population this risk does not exceed $10 \%$.

It should, however, be stressed that the quasi majority of studies on genetics and breast cancer have involved women from western countries where the incidence of this type of cancer is very high. The absence or scarcity 
of studies in Morocco and in developing countries in general, does not allow quantification of the role of these mutations in the genesis of breast cancer in our country.

In Tunisia, according to a study by Monastiri et al. [19], BRCA1 was involved in $6.25 \%$ of Tunisian women with a hereditary risk of breast cancer.

Other researchers indicated family history as a risk for breast cancer [20-22]. More precisely, Shapiro et al. noted that there were associations between risk of breast cancer and family history, low parity and high socioeconomic status [11].

According to our results, we found no relationship between family history and women who contract breast cancer $(p>0.05)$.

\subsubsection{Contraceptives and Hormones}

The possible effects of oral contraceptive hormones on the risk of breast cancer have been the subject of much research. Several authors incriminate oral contraception and alternatives to menopause. The risk is increased by early puberty, late menopause, delayed age at first pregnancy, and low parity.

In a case-control study of 419 breast cancers of women in Cape Town, South Africa, Shapiro et al. [11] found a weak association in women taking the contraceptive estrogen progesterone $(\mathrm{OR}=1.2)$. This association was slightly stronger among young women under 35 years (OR $=1.7$ ) but no association was observed with duration of use. The same study investigated the risk associated with progestin injectable contraceptives, particularly medroxyprogesterone acetate (DMPA). The results suggested that these represent only a small risk, if any, and it is limited to young women whose age is under 35 [23].

According to the Endogenous Hormones and Breast Cancer Collaborative Group [24], the use of oral contraceptives is associated with an increased risk of breast cancer in post-menopausal women in general (OR 1.19, 95\% CI, 1.09 to 1.29). In this study which provided data on nulliparous and parous women/multiparous separately, the use of oral contraceptives was associated with the risk of breast cancer among both parous women/multiparous (OR $1.29,95 \%$ CI, 1.20 to 1.40 ) and nulliparous women (OR 1.29, 95\% CI, 0.92 to 1.67). A more prolonged use of oral contraceptives among nulliparous women does not significantly modify the risk (OR 1.29, 95\% CI, 0.85 to 1.96). Among parous women/multiparous, the association was stronger when oral contraceptives were used before first term pregnancy (FTP) (OR, 1.44, 95\% CI, 1.28 to 1.62) than when they were taken after FTP (OR 1.15, 95\% CI, 1.06 to 1.26). The strongest link between the use of oral contraceptives and the risk of breast cancer was observed among parous women/multiparous who used oral contraceptives at least four years before the FTP (OR 1.52, $95 \%$ CI, 1.26 to 1.82 ).
In other respects, exposure to endogenous hormones as well as the use of exogenous hormones like oral contraceptives or hormonal treatments for menopause are associated with increased risk of breast cancer. A meta-analysis of case-control studies suggested that the use of oral contraceptives, especially at a young age (before the first pregnancy) was associated with an increased risk of pre-menopausal breast cancer [25].

Abdel-Rahman et al. dealt with 180 cases of breast cancer from two hospitals in Egypt with 192 hospital controls (without malignancy) [26]. They found that breast cancer was associated with the usual reproductive variables (nulliparity, age at first birth, age of puberty and age of natural menopause).

For other researchers, it seems that there is an increase of low but detectable risk in women taking oral contraceptives, but the risk decreases when contraception ceases, and after 10 years, no risk remains [27].

\subsection{Cervical Cancer}

Cervical cancer of the uterus is the second most common cancer among women worldwide and the first cause of female cancer deaths in developing countries [3]. In North Africa, its incidence is rather low. In Morocco, the register of cancer in the region of Casablanca (RCRC) estimated an incidence of around 35 per 100,000 in 2004. However, this type of cancer remains dominant among Moroccan women since it is ranked second after breast cancer.

In eastern Morocco and according to figures from the regional centre of Oujda (RCO), this type of cancer represents $16 \%$ of all cancers registered in women, occupying the second place after breast cancer [28].

\subsubsection{Social Determinants of Cervical Cancer}

Early observers noted the very marked differences in the risk of cervical cancer according to social determinants such as traditional social status, occupation, marital status and ethnicity. However, although these risk factors are similar to those indicated in other countries, the low socioeconomic level emerges as a particularly important factor in the North African region.

In this direction, two studies of human papillomavirus (HPV) have been published in Morocco and Algeria: The first by the National Institute of Oncology in Rabat and the second by the Centre Pierre et Marie Curie in Algiers for fight against cancer. According to these studies, one of the striking results was the illustration of the fact that the vast majority of patients with cervical cancer were illiterate or did not have access to primary education. Multiparity ( $>6$ pregnancies), age at marriage $(<16)$, age at first pregnancy $(<17)$, and the number of sexual partners $(>3)$ were all significant factors promoting cervical can- 
cer. The Moroccan study showed a strong negative association with social status, family income and educational attainment [29].

An association between low social status (as measured by literacy) and the risk of cervical cancer was also observed by Skinner et al. [30].

Consistent with the previous indications, our investigation showed that more than $3 \%$ of women with this cancer were poor, belonging to a class with a low marital status of widowed or divorced women having been married at an early age ( $<18$ years).

\subsubsection{Cervical Cancer and Hygiene}

Vaginal Hygiene is another cause. The case-control study of Bayo et al. [31] in Bamako, Mali, has shown that intimate vaginal cleaning reduces the risk of cervical cancer [OR (always versus never) $=0.06$ (95\% CI: 0.01 to 0.24 )], while use and reuse of pads constituted a high risk. The study in Morocco by Chaouki et al. [29] ended up with similar findings: a decreased risk (adjusted for age, income status and HPV) associated with washing the genitals during menstruation (OR (always vs never) $=0.26$, $95 \%$ CI 0.10 to 0.72 ), and commercial use of sanitary napkins rather than disposable towels home $(\mathrm{OR}=0.36$, $95 \%$ CI of 0.13 to 0.95 ).

\subsubsection{Cervical Cancer and Contraceptives}

Contraceptive use has been reported as a risk factor for cervical cancer. The case-control study by Chaouki et al. has shown a positive relationship between oral contraceptives and the risk of cancer of the cervix among HPV-positive women [29]. In contrast, in the case-control study in Nairobi, Kenya, which was part of the WHO collaborative study on the association between neoplasia and steroid contraceptives, the relationship was weak and not significant [32].

In our study, we found a significant association between contraceptives and cervical cancer in 5\% of women using the pill and $8 \%$ of women using injectables.

\section{CONCLUSIONS}

Breast and cervical cancers are among the most frequent and deadly in Morocco. Their burden can be reduced by early diagnosis and treatment. Unfortunately, so far these types of cancer are detected at an advanced stage in a large proportion of women. Thus, awareness, sensitization and early detection remain the preferred tracks to master these types of cancers and alleviate their socioeconomic burden.

Social determinants and risk factors are partly known and most related to the reproductive life of women and their general status. However, a part of the increase in incidence remains unexplained. The search for other environ- mental factors such as exposure to physical, chemical and biological agents is yet to achieve.

In this sense, any preventive strategy must take into account the natural history of the disease and its various stages of progress based on cultural and socioeconomic factors.

The high proportion of illiterate and poor women in our region may compromise the effectiveness of any mass screening with mammography given the additional social and financial costs. Consequently, Moroccan health authorities should seriously consider the cost-effectiveness and cost benefit of preventive strategies based on screening for early detection and treatment especially for the large population of disadvantaged women.

\section{REFERENCES}

[1] International Agency for Research on Cancer (2008) Globocan 2008: Cancer incidence and mortality worldwide in 2008. http://globocan.iarc.fr/

[2] Anne, M., Bernard, H. and Cuy, K. (2005) Nouveaux cahiers de l'infirmière. "Soins infirmiers en Cancérologie et Hémopathies”. Quatrième Édition Masson, Paris.

[3] INCa (2009) La situation du cancer en France en 2009. Collection rapports synthèses, ouvrage collectif édité par l'INCa, Boulogne Billancourt.

[4] Erik, C., Israël, N. and Nazbanou, H. (2003) Nouveaux cahiers de l'infirmière. "Soins infirmiers en maternité et aux personnes atteintes d'affections gynécologiques". Troisième Édition Masson, Paris.

[5] Boutayeb, S., Boutayeb, A., Ahbeddou, N., Boutayeb, W., Essaadi, I., Tazi, M. and Errihani, H. (2010) Estimation of the cost of treatment by chemotherapy for early breast cancer in Morocco. Cost Effectiveness and Resource Allocation, 8, 16. doi:10.1186/1478-7547-8-16

[6] RCRC (2007) Registre des cancers de la région du Grand Casablanca: Année 2004. Edition Association Lalla Salma, Rabat.

[7] Maamri, A. (2010) Screening for cervical uterine and breast cancer in a population of women 25 - 60 years in Oujda, Morocco. Proceeding of the 32nd Annual Meeting of International Association of Cancer Registries, Okouhama, 3-5 June 2010.

[8] Ben Abdallah, M., Achour, N., Saadi, A., Boussen, H., Ben Romdhane, K., Hechiche, M., Hsairi, M., Maalej, M., Zehani, S. and Ben Ayed, F. (2009) Cancer du sein en Tunisie: Caractéristiques épidémiologiques et tendance évolutive de l'incidence. Tunis-Medindustrie, 87, 417425.

[9] Boutayeb, A. and Boutayeb, S. (2005) The burden of non communicable diseases in developing countries. International Journal for Equity in Health, 4, 2. doi:10.1186/1475-9276-4-2

[10] Afsse, T. (2011) Étude d'impact sur les coûts que représentent pour l'assurance maladie certaines pathologies liées à la pollution. 
http://www.afssa.fr/ET/DocumentsET/impact_sanitaire_p ollution environnemental.pdf

[11] Shapiro, S., Rosenberg, L., Hoffman, M., Truter, H., Cooper, D., Rao, S., Dent, D., Gudgeon, A., van Zyl, J., Katzenellenbogen, J. and Baillie, R. (2000) Risk of breast cancer in relation to the use of injectable progestogen contraceptives and combined estrogen/progestogen contraceptives. American Journal of Epidemiology, 151, 396403.

[12] Kogevinas, M., Pearce, N., Susser, M. and Boffetta, P. (1997) Social inequalities and cancer. IARC Scientific Publications, Lyon.

[13] Kato, I., Miura, S., Kasumi, F., Iwase, T., Tahiro, H. and Fujita, Y. (1992) A case-control study of breast cancer among Japanese women: With special reference to family history and reproductive and dietary factors. Breast Cancer Research Treatment, 24, 51-59. doi:10.1007/BF01832358

[14] Boutayeb, A. (2011) Social determinants of reproductive health in Morocco. African Journal of Reproductive Health, 15, 57-66.

[15] Sakka, F. and Chelly, H. (2000) Age et vécu de la pubertéchez la jeune fille tunisienne. Tunis-Medindustrie, 78, 191-194.

[16] The Breast cancer linkage consortium (1999) Cancer risks in BRCA 2 mutation carriers. Journal of the $\mathrm{Na}$ tional Cancer Institute, 91, 1310-1316.

[17] Collaborative Group on Hormonal Factors in Breast Cancer (2001) Familial breast cancer: Collaborative reanalysis of individual data from 52 epidemiological studies including 58209 women with breast cancer and 101986 women without the disease. Lancet, 27, 13891399.

[18] Sasco, A.J. (2003) Breast cancer and the environment. Hormone Research, 60, 50-59. doi:10.1159/000074500

[19] Monastiri, K., Ben Ahmed, S., Presneau, N., Bignon, J.Y. and Chouchane, L. (2002) Dépistage rapide des mutations germinales du gène BRCA1 par le test de la proteine tronquée chez les familles tunisiennes. Tunis-Medindustrie, 80, 515-518.

[20] Lambe, M. and Hsieh, C.C. (1997) Parity, age at first birth and risk of breast cancer: A population based study in Sweden. Cancer Research Treatment, 38, 305-311. doi:10.1007/BF01806150

[21] Freudenheim, J.L. and Marshall, J.R. (1997) Lactation history and breast cancer risk. American Journal of Epidemiology, 146, 932-938.

[22] INSERM (2008) Cancers et environnement, une expertise collective. Edition INSERM, Paris.

[23] Skegg, D.C.G., Paul, C., Spears, G.F.S. and Williams, S.M. (1996) Progestogen-only oral contraceptives and risk of breast cancer in New Zeland. Cancer Causes Control, 7, 513-515. doi:10.1007/BF00051883

[24] The Endogenous Hormones and Breast Cancer Collaborative Group (2002) Endogenous sex hormones and breast cancer in postmenopausal women: Reanalysis of nine prospective studies. Journal of the National Cancer Institute, 94, 606-616. doi:10.1093/jnci/94.8.606

[25] The Endogenous Hormones and Breast Cancer Collaborative Group (2011) Circulating sex hormones and breast cancer risk factors in postmenopausal women: Reanalysis of 13 studies. British Journal of Cancer, 105 709-722. doi:10.1038/bjc.2011.254

[26] Abdel-Rahman, H.A., Mostafa, R., Shoulah, A.R., Wassif, O.M., Sallih, M.A., El Gendy, S.D. and Abdo, A.S. (1993) An epidemiological study of cancer breast in greater Cairo. Journal of Egyptian Public Health Association, 68, 119-142.

[27] Reeves, G. (1996) Breast cancer and oral contraceptivesThe evidence so far. Cancer Causes Control, 7, 495-496. doi:10.1007/BF00051880

[28] Maamri, A., Mbarki, F., Chafi, A. and El Hfid, M. (2010) Dépistage du cancer du sein et du col de l'utérus chez une population de femmes âgées entre 25 et 60 ans. Proceeding of the 8th congress of the International Association of Epidemiology, Beyrouth, 21-23 May 2010, 22-33. doi:10.1002/(SICI)1097-0215(19980209)75:4<546::AIDIJC9>3.0.CO;2-T

[29] Chaouki, N., Bosch, F.X., Munoz, N., Meijer, C.J.L.M., el Gueddari, B., el Ghazi, A., Deacon, J., Castellsague, X. and Walboomers, J.M.M. (1998) The viral origin of cervical cancer in Rabat, Morocco. International Journal of Cancer, 75, 546-554.

[30] Skinner, M.E.G., Parkin, D.M., Vizcaino, A.P. and Ndhlovu, A. (1993) Cancer in the African population of Bulawayo, Zimbabwe, 1963-1977. IARC Technical Report, Lyon.

[31] Bayo, S., Parkin, D.M., Koumare, A.K., Diallo, A.N., Ba, T., Soumare, S. and Sangare, S. (1990) Cancer in Mali: 1987-1988. International Journal of Cancer, 45, 679-684. doi:10.1002/ijc.2910450418

[32] Williams, M.A., Kenya, P.R., Mati, J.K.G. and Thomas, D.B. (1994) Risk factors for invasive cervical cancer in Kenyan women. International Journal of Epidemiology, 32, 906-912. doi:10.1093/ije/23.5.906 